\title{
Epitrochlear Lymph Node Dissection
}

\author{
Steven N. Hochwald, MD, Nicole Kissane, MD, Stephen R. Grobmyer, MD, and James Lopes, MD \\ Division of Surgical Oncology, Department of Surgery, University of Florida College of Medicine, Gainesville, FL
}

\begin{abstract}
Epitrochlear node involvement occurs in a small minority of patients with forearm or hand melanoma. Although in-transit sentinel lymph nodes are identified infrequently, they contain metastatic disease at nearly the same frequency as sentinel lymph nodes in cervical, axillary, and inguinal nodal basins. Positive in-transit sentinel lymph nodes are likely to be the only site of nodal metastasis. Therefore, detailed preoperative lymphoscintigraphy and meticulous intraoperative search for in-transit
\end{abstract}

nodes should be performed. The recovery of nodes from intransit nodal areas is low; however, there appears to be an increase in the performance of these dissections since the advent of lymphatic mapping and sentinel lymph node biopsy. This streaming video demonstrates the incidence of epitrochlear lymph node involvement and technical considerations associated with epitrochlear lymph node dissection.
Electronic supplementary material The online version of this article (doi:10.1245/s10434-010-1227-5) contains supplementary material, which is available to authorized users.

(C) Society of Surgical Oncology 2010

First Received: 1 October 2009;

Published Online: 3 August 2010

S. N. Hochwald, MD

e-mail: steven.hochwald@surgery.ufl.edu 